\title{
Perception: A HyperCard stack for demonstrating visual perceptual phenomena
}

\author{
ISMAEL E. CARRERAS \\ Bates College, Lewiston, Maine
}

\begin{abstract}
Perception is a HyperCard stack that allows users to explore visual illusions and other perceptual phenomena on the Apple Macintosh. The stack contains over 20 demonstrations of intersecting line illusions, size and shape illusions, subjective contours, color assimilation, and so forth. As a presentation tool for classroom or laboratory demonstrations, Perception offers three unique features for displaying visual phenomena: (1) the capability to "dissolve" the inducing elements of an illusion in order to show the objective state of affairs, (2) the ability to quickly reverse the inducing elements of an illusion and therefore the effects of the distortion, and (3) animation of the various components of an illusion to produce continuous distortions in real time. These features are illustrated with use of the Orbison, Titchener, Hering, and Wundt illusions. Use of the stack reveals two interesting and unanticipated findings: (1) an apparent size distortion in the central square of the Orbison illusion as it moves back and forth across the background of concentric rings, and (2) perceptual aftereffects that arise when the inducing elements of the Titchener, Hering, or Wundt illusion is dissolved.
\end{abstract}

Perception is a HyperCard stack (Atkinson, 1987) that students use to explore various types of visual phenomena. This stack originated with the author's undergraduate thesis, which was about the effects of computer animation on the appearance of subjective contours (Carreras, 1990). In that study, subjects were shown transparent subjectivecontour figures adapted from Gerbino and Kanizsa (1987). On an IBM-compatible microcomputer, the inducing elements of the contours were manipulated to produce either a stationary or a moving subjective contour figure. The subjects rated the clarity of the contours and the color intensity of the transparent figure. ${ }^{1}$ One offshoot of that project was the idea of utilizing a microcomputer to display visual illusions in a "dynamic" fashion; that is, computer animation would be used to produce, eliminate, and continuously alter the distortion associated with a particular visual illusion. A tacit assumption motivating this project is that computer animation can reveal the underlying dynamics of an illusory phenomenon more effectively than the traditional "static" displays employed in textbooks on visual perception.

The author decided to use HyperCard as the software medium because of its low cost, widely installed user base, effective scripting environment, and easy-to-use painting tools. Various optical illusions were created, and scripts were developed that allowed the user to manipulate the

The author would like to thank Drake $\mathbf{R}$. Bradley for encouraging submission of this paper, as well as for his critical comments and suggestions on a first draft of this paper and for performing the beta testing of the Perception software. Correspondence should be addressed to I. E. Carreras, Bates College, 23 Campus Avenue, Lewiston, ME 042406058 (e-mail: icarreras@adm.bates.edu). direction, presence, or absence of the inducing elements, and/or to set parts of the illusory display into motion. It was reasoned that the dynamics of each illusion would be more effectively illustrated if a consistent user interface was employed across the various demonstrations. This was accomplished with three methods of manipulation. First, a dissolve button is provided, which removes the inducing elements of the illusion to reveal the objective or "true" state of affairs. Clicking this button removes the fins of the Müller-Lyer illusion, the outside circles of Titchener's circles illusion, the background rings of the Orbison illusion, and so forth. Second, a reverse button allows the user to reverse the direction of the inducing elements of the illusion and thereby instantaneously reverse the direction of the illusory distortion. Clicking this button flips the fins of the Müller-Lyer illusion, interchanges the outside circles of Tichener's circles illusion, and so on. Finally, a motion button is provided, which sets the "target" figure of an illusion into motion against the background. Clicking this button sets the central square of the Orbison illusion, the parallel lines of the Hering and Wundt illusions, and so on, into motion over the background grid. This permits the user to view the illusory distortion as it unfolds in real time.

The use of HyperCard and other programs to conduct research and to demonstrate perceptual phenomena on the Macintosh is not new, as is illustrated by stacks such as Doherty's (1990) Contour, and by sophisticated programs such as MacLab (Chute, 1986) and VSearch (Enns, Ochs, \& Rensink, 1990). A major distinction between Perception and other software packages is that active manipulation of the illusions is a central and consistent feature of the user interface. Furthermore, Perception's cost is nominal in comparison with that of other software packages. 

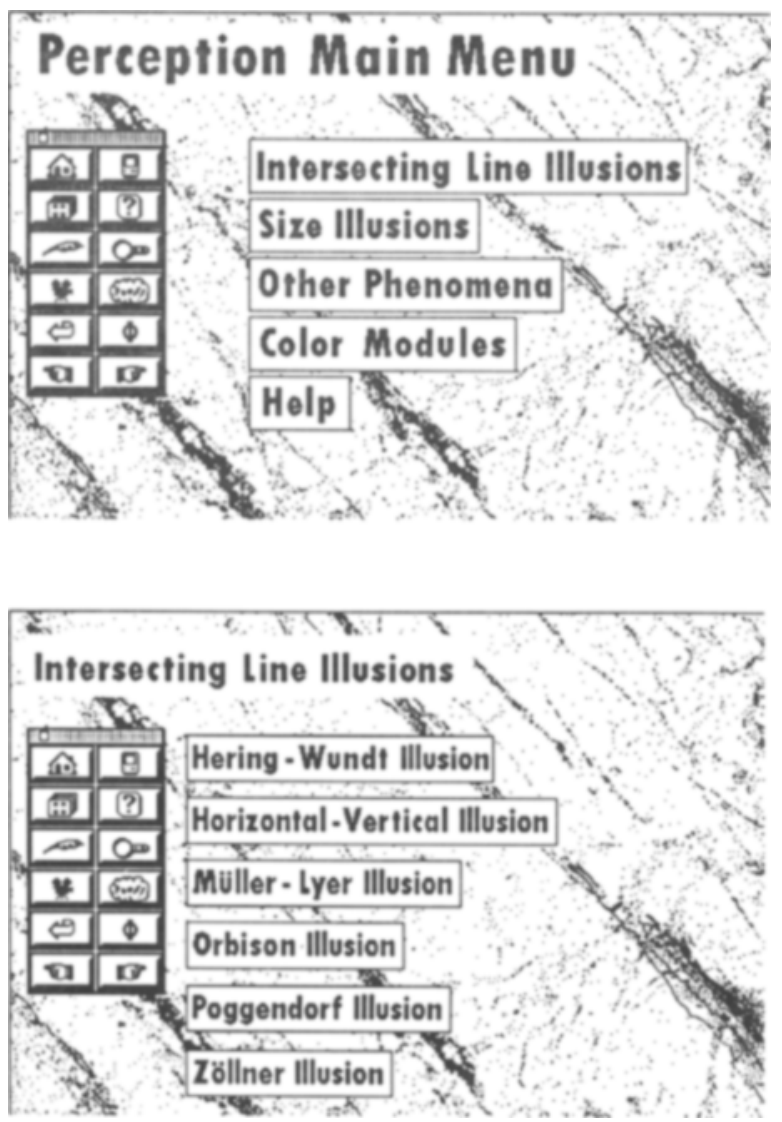

Figure 1. Perception Main Menu card (top). Category card with related illusion modules (bottom).

\section{DESCRIPTION OF THE PROGRAM}

The stack contains demonstrations of intersecting line illusions, size and shape illusions, subjective contours, and color assimilation and contrast. As shown in Figure 1 (top panel), Perception's Main Menu screen has five buttons naming major categories: Intersecting Line Illusions, Size Illusions, Other Phenomena, Color Modules, and Help. By pressing one of the category buttons on the Main Menu, the user accesses another card containing buttons with the names of the demonstration modules in that category. Figure 1 (bottom panel) shows the demonstration modules for the intersecting line illusions. Clicking one of these buttons loads the corresponding demonstration.

It is a good idea to select Help on first viewing Perception, because it explains in detail the control palette functions shown on the left side of Figure 1 (bottom panel). The control palette unifies the interface of the program; its buttons are consistently available across the demonstrations. The upper six buttons in the palette are used to go to the Home Card, exit to the Finder, return to the main menu in Perception, invoke on-line help, obtain information about the author and the program, and display information about the particular illusion being viewed. The lower six buttons in the palette allow the user to manipulate the illusions in various ways. The "Poof!" button dissolves (and restores) the inducing elements of the illusion, the movie camera button starts (and stops) an animation, the reversearrow button reverses the inducing elements, and the $\phi$ (for phi) button moves a target in discrete rather than continuous displacements over a background. Finally, the bottom two buttons, with hands pointing left and right, are used to move backward or forward in the stack of cards illustrating the variants of a particular visual illusion. When a particular illusion module is loaded, the icons of the buttons that may be used to manipulate the illusion are displayed in the lower left corner of the card. This is illustrated in Figure 2 (top panel) for the Orbison illusion: the icons indicate that the dissolve, animation, and phi buttons are all active for the Orbison illusion. Furthermore, the left- and right-hand icons indicate that other variants of the Orbison illusion are available. Clicking the righthand button, for example, displays an Orbison illusion with a diamond rather than a square in the center of the figure.

Many observers viewing the central square of the Orbison illusion in Figure 2 (top panel) find it difficult to believe that the sides are really straight. Clicking on the dissolve button causes the background of concentric rings to disappear, as in Figure 2 (bottom panel), and shows that the sides of the square are, in fact, straight. Clicking the

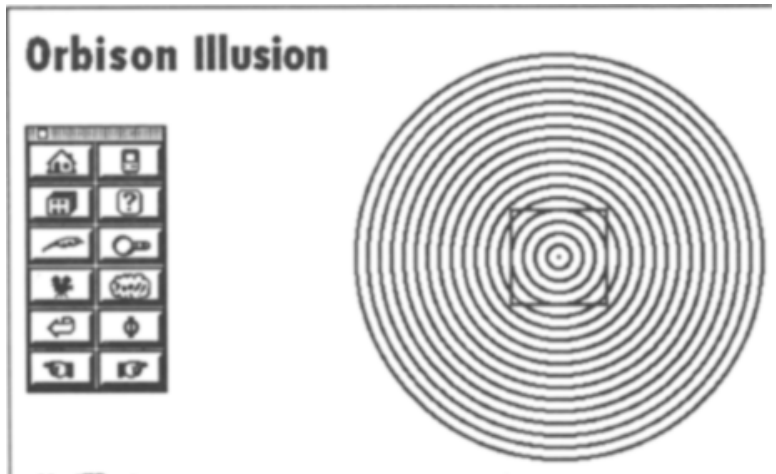

\section{Orbison Illusion}

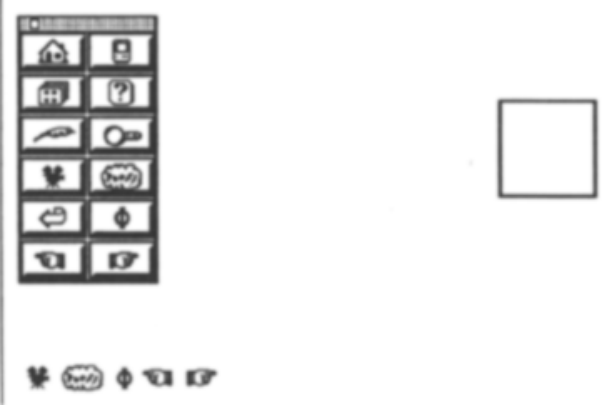

Figure 2. Orbison Illusion (top). Orbison Illusion with dissolve feature activated (bottom). 


\section{Hering Illusion}
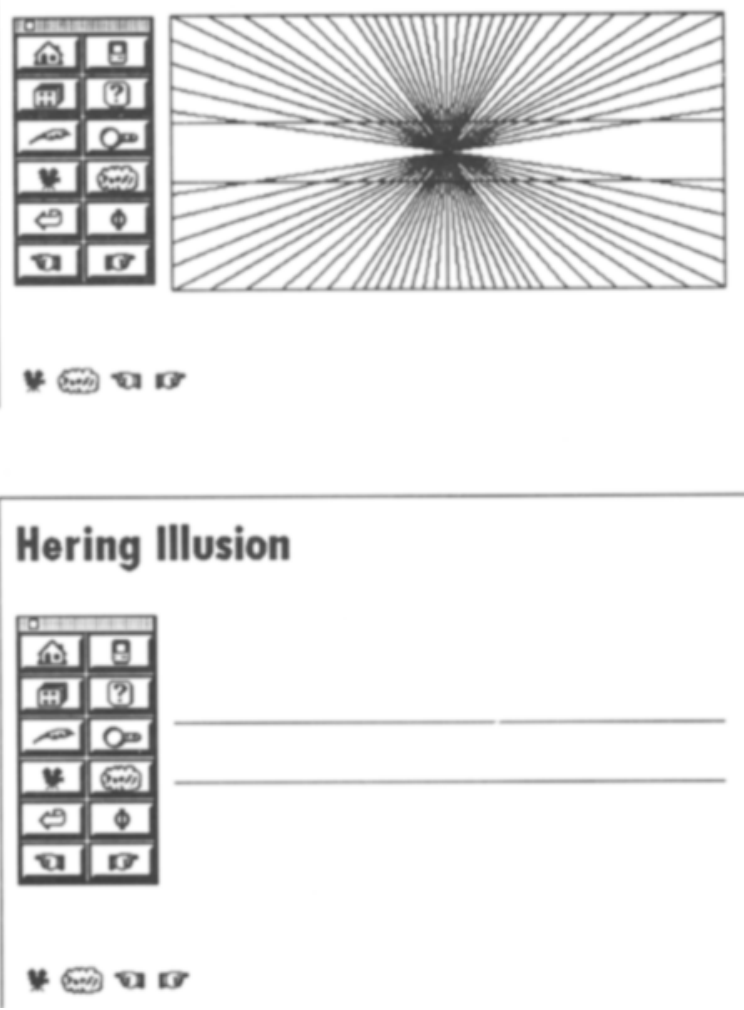

Figure 3. Hering Illusion (top). Hering Illusion with dissolve feature activated (bottom).

dissolve button a second time restores the background, and as the concentric rings reappear, the sides of the square appear to bow inward again. As another example of an intersecting line illusion, consider the Hering illusion displayed in Figure 3 (top panel). The radial lines emanating from the center of the figure distort the parallel lines so that they appear to bulge outward from the center. Figure 3 (bottom panel) illustrates how these lines look when the background field is hidden by clicking the dissolve button. This same approach is employed with size illusions, as illustrated in Figure 4 (top panel) for Titchener's circles illusion. Although the center circle on the left appears smaller than the center circle on the right (possibly because of size contrast with the outer circles), activating the dissolve button shows that the two circles actually have the same size (Figure 4, bottom panel). Note that the dissolve button works by removing the inducing elements that are responsible for the illusion. For the shape distortions produced by intersecting line illusions, this means removing the background grid. For size distortions, it means removing the specific elements-the outside circles in Tichener's illusion, the fins in the Müller-Lyer illusion, the converging lines in the Ponzo illusion, and so on-that cause the illusion. In all these cases, the observer is able to elimi- nate and recreate the illusion at will by simply toggling the dissolve button.

One other use of the dissolve button is to provide visual cues. Figure 5 (top panel) shows a well-known example of a hidden figure. An observer who has not seen this figure before may not see the cow immediately, or even after several minutes. Clicking the dissolve button provides the necessary feedback for one to successfully organize the figure. In this case, the dissolve button works in reverse: "dissolving" the cues so that in Figure 5 (bottom panel), the subject sees not only the gray outline denoting the cow, but also hears a "Moo!" sound at the same time. Clicking the dissolve button a second time restores the original figure, and most observers now have no difficulty seeing the cow. This helps to demonstrate the all-or-none character of perceptual learning for hidden figures, Street figures, ambiguous figures, and the like: once a possible organization has been achieved through selective prompting, it proves to be highly resistant to forgetting.

The second method by which the user interacts with a visual illusion is provided by the reverse button. For illusions whose inducing elements can be reversed or exchanged, activating the reverse button will perform this operation. Figure 6 (top panel) shows a Müller-Lyer figure in

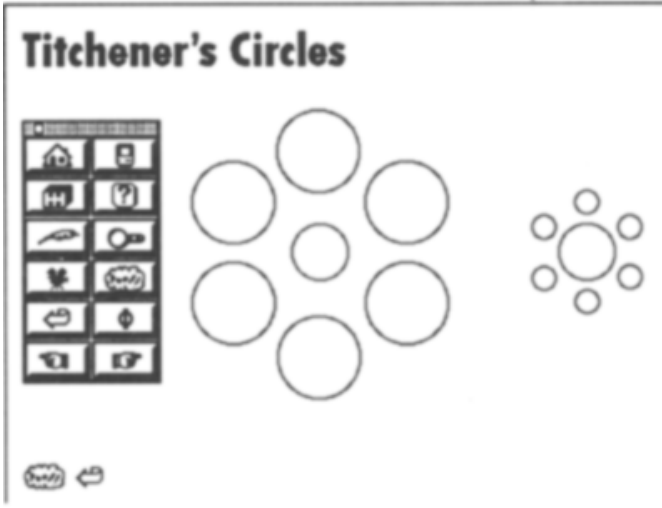

\section{Titchener's Circles}
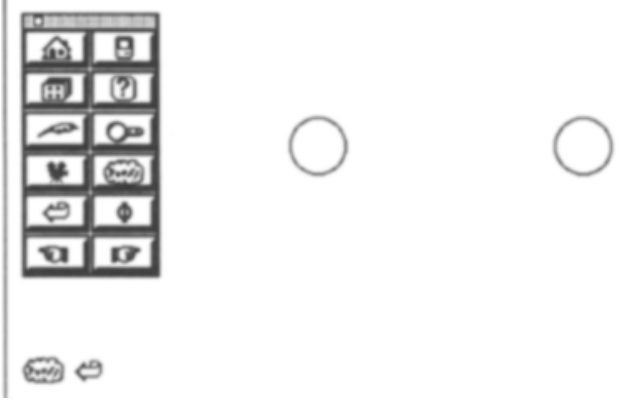

Figure 4. Titchener's Circles (top). Titchener's Circles with dissolve feature activated (bottom). 

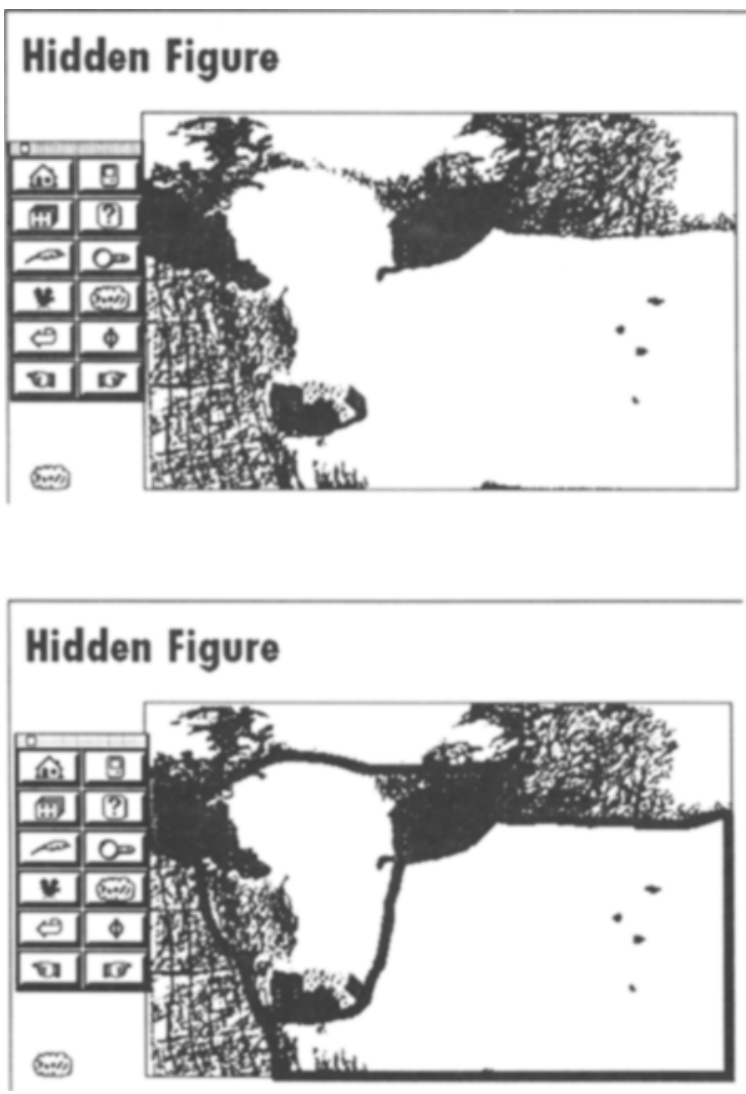

Figure 5. Hidden Figure (top). Hidden Figure with dissolve feature activated (bottom).

which the top horizontal shaft is bounded by ingoing fins and the bottom shaft by outgoing fins. This causes the top shaft to appear shorter than the bottom shaft. Clicking the reverse button reverses the direction of the fins, as shown in Figure 6 (bottom panel), which reverses the apparent size distortion as well. By clicking the reverse button several times in succession, the observer can watch the horizontal shafts alternately grow and shrink. Clicking on the dissolve button removes the fins altogether and demonstrates that the horizontal shafts have the same length.

As another example, consider the Delboeuf illusion in Figure 7 (top panel). This figure shows two pairs of concentric circles. The outside circle on the left, labeled "A," has the same size as that of the inside circle on the right, labeled "B." However, since "A" becomes assimilated to the smaller circle inside it, and " $B$ " becomes assimilated to the larger circle outside it, most observers perceive " $A$ " as being smaller than " $B$." Clicking the reverse button exchanges the concentric rings so that " $A$ " is now surrounded by the larger circle, and " $B$ " now surrounds the smaller circle. This reverses the apparent size distortion, so that " A" now appears to be larger than "B."

In the Tichener's circles illusion presented earlier in Figure 4 (top panel), clicking the reverse button reverses the location of the large and small circles surrounding the center circles, which in turn reverses the apparent size distortion. Successively clicking the reverse button causes the center circles to alternately grow and shrink as the outside circles exchange positions.

As noted above, animation is used to produce several interesting effects. For example, many observers find it difficult to see illusory contours in Figure 8 (top panel) when first shown the figure. However, as soon as the "cube" is set in motion by clicking the animate button, a phenomenally complete three-dimensional Necker cube is seen moving back and forth over eight black disks and a white background (Figure 8, bottom panel). The illusory contours delineating the bars of the cube are seen to sweep back and forth over an otherwise uniform background. Bradley and Lee (1982) report data showing that setting a subjective contour figure in motion relative to the background serves to strengthen the clarity and vividness of the contours.

The subjective Necker cube in Figure 8 (top panel) is subject to several alternative perceptual organizations, some of which are quite dramatic. In addition to the cube-infront organization just described, there is a cube-in-back organization: each corner of the Necker cube is seen through a "hole" punched in a white surface (or slice of "swiss cheese"), and the entire cube appears to be sus-

\section{Müller - Lyer Illusion}
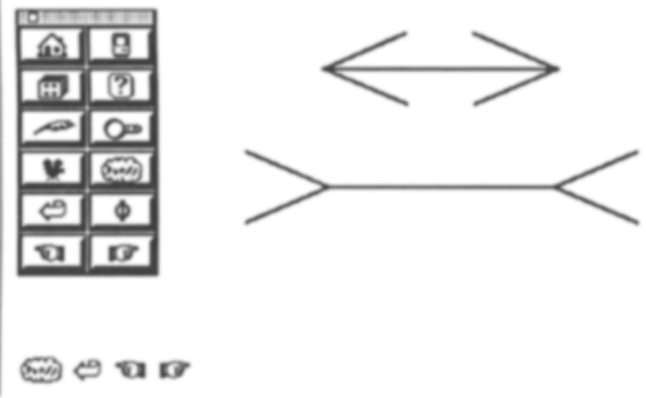

\section{Müller - Lyer Illusion}
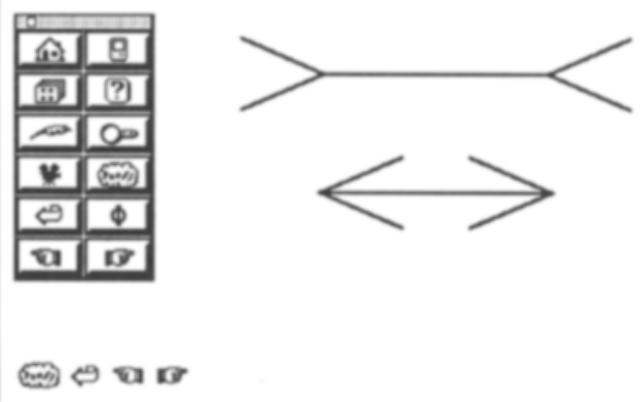

Figure 6. Müller-Lyer Mlusion (top). Müller-Lyer Illusion with reverse feature activated (bottom). 


\section{Delboeuf Illusion}
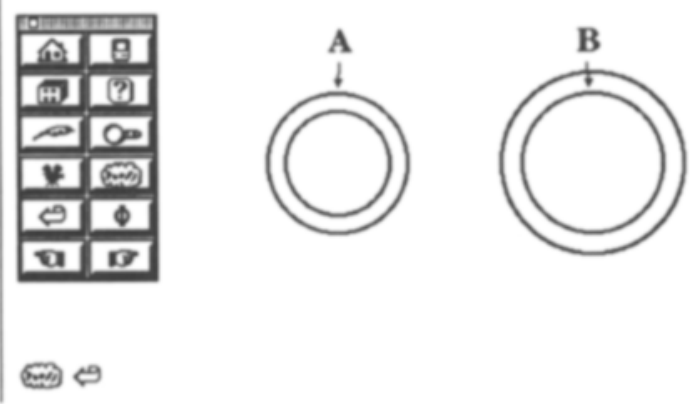

Delboeuf Illusion
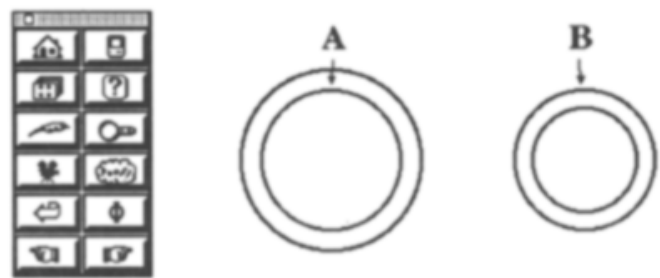

$\Leftrightarrow$

Figure 7. Delboeuf Illusion (top). Delboeuf Ilusion with reverse feature activated (bottom).

pended in a dark enclosure. ${ }^{2}$ Illusory contours are now seen delineating the interior edges of the "holes" rather than the edges of the bars. It is also possible to achieve a "straddled" organization, with one face of the cube in front and one face in back. Cards in the subjective Necker cube stack provide animations illustrating each of these organizations. In one animation, as the "cube" sweeps back and forth, it occludes a speckled gray pattern drawn between the black disks, thus forcing a cube-in-front organization. In a second animation, the moving "cube" fails to occlude gray spots placed between the disks, which forces a cube-inback organization. A third animation combines these occlusion cues so that the lower face of the cube is seen in front, whereas the upper face is seen in back. In each case, subjective contours are interpolated wherever necessary, in order to demarcate the edges of surfaces that are perceived to occlude other surfaces. The various animations provide a fairly convincing demonstration of the effects of perceptual organization on the appearance of subjective contours (Bradley, 1987).

The Perception stack also uses animation to illustrate the continuous distortion of a figure as it moves across a back- ground. Consider the Orbison illusion shown in Figure 9 (top panel): clicking the animate button causes the central square to move back and forth across the field of concentric rings. As shown in Figure 9 (bottom panel), the apparent shape of the square is quite different when it is located at the periphery of the display. When at the center, all four sides of the square appear to bend inward, producing a "pincushion" distortion. When at the periphery, the left and right sides appear to bend in the same direction (left), the top and bottom sides appear to be straight but diverging, and the overall effect is that of a "trapezoid.", As the square moves back and forth during the animation, its shape continuously deforms and changes. This provides a very dramatic demonstration of the effects of intersecting lines on the perceived shape of an object. Another example is provided by the Wundt illusion. As shown in Figure 10 (top panel), when located in the center, the parallel lines appear to bow inward (toward the center), but as they move to the top of the display (Figure 10, bottom panel) both lines appear to bend upward. Once again, the lines are seen to con-

\section{Subjective Necker Cube}

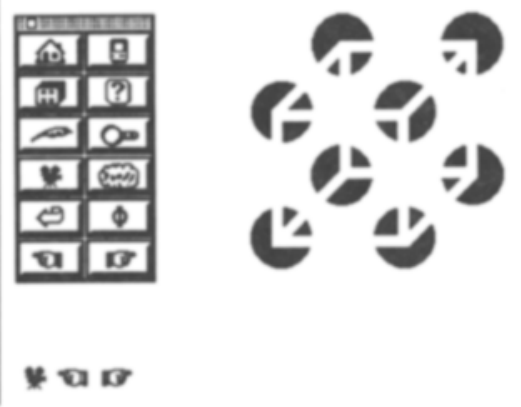

\section{Subjective Necker Cube}
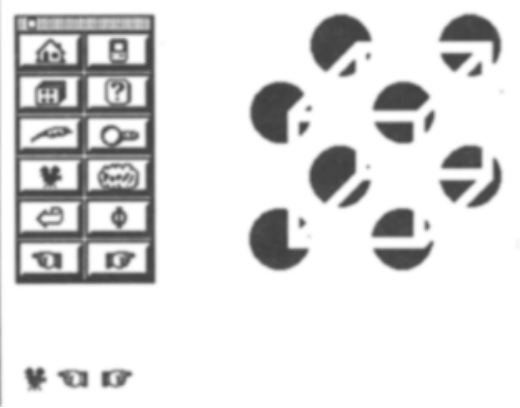

Figure 8. Subjective Necker Cube-stationary cube in center position (top). Subjective Necker Cube-cube in motion at extreme right position (bottom). 

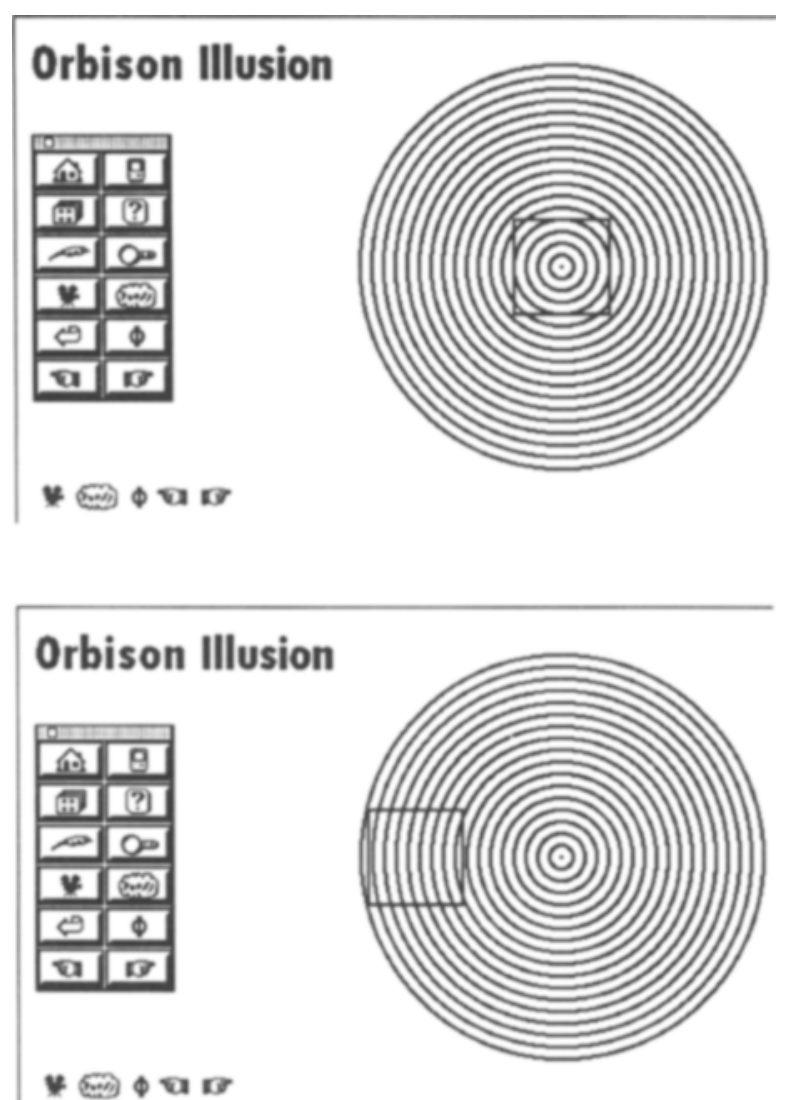

Figure 9. Orbison Illusion-geometric figure in center of background field (top). Orbison Illusion-geometric figure at the edge of background field (bottom).

tinuously become distorted as they sweep back and forth across the background.

\section{UNANTICIPATED OUTCOMES}

As the software was being used to demonstrate visual illusions, two unanticipated perceptual effects were encountered. In the animation of the Orbison illusion described above (Figure 9), many observers reported that the square appeared to grow larger as it moved from the center to the periphery of the concentric rings. This apparent size distortion was subsequently verified in an experiment conducted by True (1990). This would seem to be the first indication that the Orbison illusion is an illusion of size as well as shape.

The other unanticipated perceptual effect was encountered in the use of the dissolve button. When this button is clicked to remove the inducing elements of an illusion, a perceptual "aftereffect" is sometimes seen. With the Wundt illusion of Figure 10 (top panel), for instance, the parallel lines may appear to bend outward (away from the center) for a brief moment immediately after the background has dissolved. Similarly, the square in the Orbison figure (Figure 9, top panel) may appear to bow outward momentarily when the concentric rings are removed. In addition to these shape aftereffects, size aftereffects have also been encountered. Consider Tichener's circles illusion in Figure 4 (top panel): when the dissolve button is used to eliminate the surround circles, the circle on the left appears larger than the circle on the right for a brief moment. A similar size aftereffect is apparent with the Delboeuf illusion shown in Figure 7 (top panel), with Circle A appearing larger than Circle B following the dissolve. Now, in each of these cases, the direction of the aftereffect is opposite that of the original distortion, so these are all examples of negative perceptual aftereffects. However, in contrast to perceptual aftereffects induced by fixating an inspection stimulus such as a curved line, the "aftereffects"' noted here occur in response to purely subjective distortions: the parallel lines in the Wundt illusion only appear to be curved. That they induce negative perceptual aftereffects in subsequently viewed straight lines is certainly intriguing, and it merits further investigation.
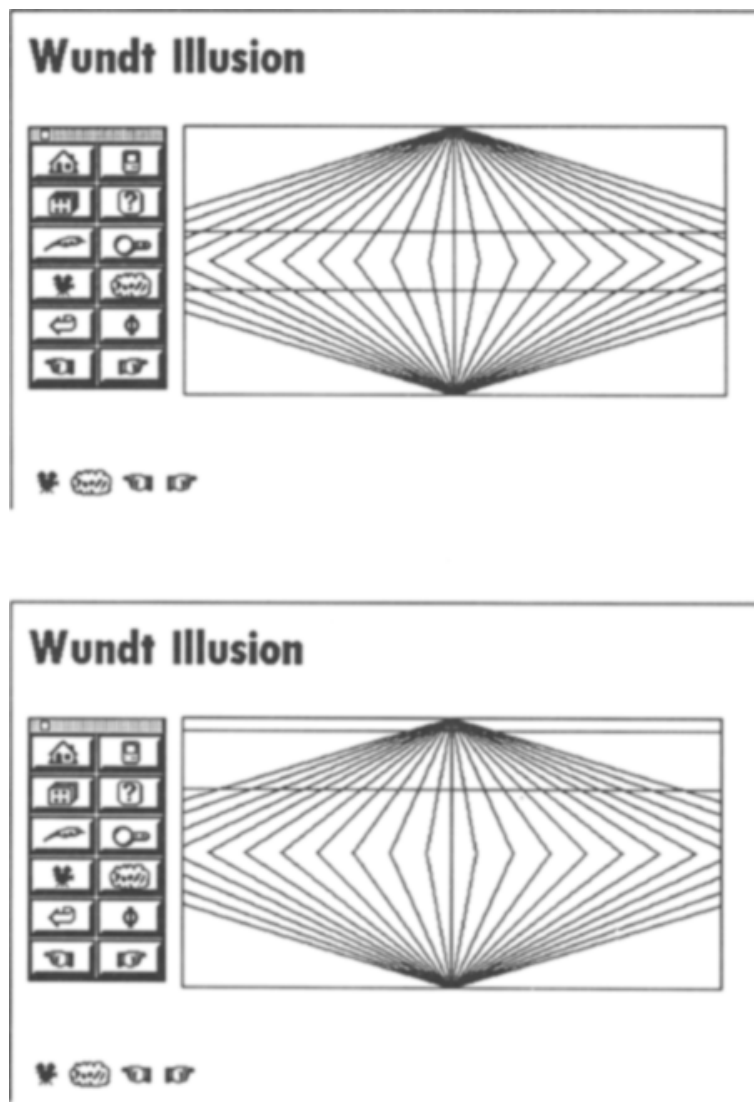

Figure 10. Wundt Illusion - parallel lines in center of background field (top). Wundt Illusion - parallel lines at the top of background field (bottom). 


\section{SYSTEM REQUIREMENTS}

Perception's minimum hardware requirements are a 2-MB Macintosh Plus with an $800 \mathrm{~K}$ drive. It is highly recommended, however, to run Perception from a hard disk in order to view smooth animation and transition sequences. Perception was created under HyperCard 2.1, which does not allow the user to run the stack under earlier HyperCard versions (1.2 or earlier). In order to view the color modules, the user will need to run Perception on a color Macintosh (a color-enhanced SE or SE/30, or a Mac II or higher).

\section{REFERENCES}

Atkinson, B. (1987). HyperCard [Computer program]. Cupertino, CA: Apple Computer.

BRADLEY, D. R. (1987). Cognitive contours and perceptual organization. In S. Petry \& G. Meyer (Eds.), The perception of illusory contours (pp. 201-212). New York: Springer-Verlag.

Bradley, D. R., \& Lee, K. (1982). Animated subjective contours. Perception \& Psychophysics, 32, 393-395.

CARRERAS, I. E. (1990). Animated chromatic subjective contours. Unpublished senior thesis, Bates College, Lewiston, ME.
Chute, D. L. (1986). MacLaboratory for psychology: General experimental psychology with Apple's Macintosh. Behavioral Research Methods, Instruments, \& Computers, 18, 205-209.

Dougherty, T. D. (1990). Contour: A hypermedia environment for teaching about subjective contours and other visual illusions. $B e$ havioral Research Methods, Instruments, \& Computers, 22, 223-227.

EnNs, J. T., OChs, E. P., \& Rensink, R. A. (1990). VSearch: Macintosh software for experiments in visual research. Behavioral Research Methods, Instruments, \& Computers, 22, 118-122.

Gerbino, W., Kanizsa, G. (1987). Can we see constructs? In S. Petry \& G. E. Meyer, (Eds.), The perception of illusory contours (pp. 246-252). New York: Springer-Verlag.

TrUE, R. (1990). Apparent size distortion in the Orbison illusion. Unpublished senior thesis, Bates College, Lewiston, ME.

\section{NOTES}

1. The results indicated that although motion increased the clarity of the subjective contours, it had relatively little effect on color intensity.

2. Perhaps for this reason, the cube in back appears substantially brighter than the cube in front.

3. These shape distortions can be derived from the assumption that the acute angles of intersecting lines are underestimated to some extent. Consequently, the sides of the square are bent or rotated to increase the acute angles formed by the concentric rings that intersect them. 